Clío América/ Vol. 13, No. 25 - 2019 / 232 - 242

DOI: http://dx.doi.org/10.21676/23897848.3025

\title{
FACTORES QUE EXPLICAN EL COMPORTAMIENTO DEL MERCADO ACCIONARIO MEXICANO
}

\section{FACTORS DRIVING THE MEXICAN STOCK ECHANG}

\author{
Gladys Iveth López-Juárez ${ }^{1}$ (i) , Rogelio Ladrón de Guevara-Cortés ${ }^{2}$ ii) y Rosa Marina Madrid-Paredones ${ }^{3}$ (i)
}

${ }^{1}$ Universidad Veracruzana, México.Email:g.la_1355@hotmail.com

${ }^{2}$ Universidad Veracruzana, México. Email: roladron@uv.mx

${ }^{3}$ Universidad Veracruzana, México. Email: rmadrid@uv.mx

Para citar este artículo: López-Juárez, G. I., Ladrón de Guevara-Cortés, R. y Madrid-Paredones, R. M. (2019). Factores que explican el comportamiento del mercado accionario mexicano. Clío América, 13(25), 232-242. doi:

http://dx.doi.org/10.21676/23897848.3025

Recibido: 29 enero de 2019

Aceptado: 21 de marzo de 2019

Publicado en línea: mayo 24 de 2019

Palabras clave:

Mercado

accionario;

índice de precios

y cotizaciones;

tipo de cambio;

tasa de interés;

precio del

petróleo.

JEL: G10; C13; E44.

Keywords:

Stock market; prices and quotation Index; exchange rate; interest rate; oil price.

\section{RESUMEN}

El objetivo de la investigación es proporcionar evidencia empírica preliminar sobre un conjunto de variables macroeconómicas que pudieran explicar el comportamiento del mercado accionario en México. La variable a explicar se encuentra representada por el Índice de Precios y Cotizaciones de la Bolsa Mexicana de Valores (IPC) y las variables macroeconómicas explicativas están conformadas por: el tipo de cambio peso mexicano-dólar, la tasa de interés y el precio del petróleo. La metodología de análisis de esta investigación empírica incluye, primero, un estudio correlacional de las variables objeto de estudio y posteriormente, un contraste unifactorial (regresión lineal simple) y multifactorial (regresión lineal múltiple) de los indicadores mencionados. Se aplica una metodología econométrica de corte transversal de datos históricos reales mensuales, que permite determinar de manera inicial la importancia e influencia de estos factores en el IPC. Los resultados obtenidos en esta investigación proporcionan evidencia empírica de la influencia que tienen dos de las variables en el comportamiento del principal índice bursátil de la Bolsa Mexicana de Valores (BMV): el tipo de cambio y el precio del petróleo.

\section{ABSTRACT}

The purpose of this research is to provide preliminary empirical evidence about a set of macroeconomic variables that could explain the behavior of the stock market in Mexico. The variable to explain is represented by the Prices and Quotations Index (IPC by its acronym in Spanish), and the explanatory variables are characterized by the exchange rate Mexican peso - United States dollar, the interest rate and the oil price. The methodology for the empirical analysis includes, first, a correlational study on the variables object of this research; and secondly, univariate (simple linear regression) and multivariate (multiple linear regression) cross-section econometric contrasts which are applied on the aforementioned indicators. The results of this research provide empirical evidence about the influence of two of these macroeconomic variables on the behavior of the Mexican Stock Exchange (BMV by its acronym in Spanish): the exchange rate and the oil price. 


\section{INTRODUCCIÓN}

El objetivo de este trabajo es proporcionar evidencia empírica preliminar sobre un conjunto de variables macroeconómicas que pudieran explicar el comportamiento del mercado accionario en México. La metodología utilizada para proporcionar dicha evidencia empírica se realiza a través de un contraste econométrico de corte transversal univariante y multivariante, utilizando datos históricos reales, para llegar a una conclusión preliminar que permita definir la importancia e influencia de estos en el comportamiento del IPC.

Los resultados de esta investigación aportan evidencia empírica sobre la existencia de una correlación significativa entre las variables explicativas y el IPC, así como una influencia por parte de dos de las variables propuestas sobre el IPC: el tipo de cambio y el precio de petróleo.

El resto de este documento se divide de la siguiente manera: primero, se describe de manera general cada una de las variables utilizadas y se hace una revisión de la de la literatura más relevante acerca de la relación y causalidad de las variables de este estudio. A continuación, se explica la metodología empleada y, después, se presentan los resultados del análisis estadístico y econométrico. Posteriormente, se plantean la discusión y conclusión de esta investigación y, finalmente, se incluyen las referencias utilizadas.

\section{Variables Macroeconómicas}

El enfoque de este trabajo responde a un interés por probar, de manera preliminar, de forma empírica la relación que tienen una serie de variables económico-financieras con el principal indicador de la Bolsa Mexicana de Valores de México. A continuación, se describen brevemente cada una de las variables y el rol de estas en nuestro modelo.

Como variable dependiente se utilizó el Índice de Precios y Cotizaciones (IPC), que es definido por la Bolsa Mexicana de Valores (2014) como el índice de capitalización del mercado accionario en México, calculado para representar de manera ponderada el comportamiento y las variaciones del mercado accionario a través de una muestra en un momento determinado.

Como variables independientes, primero, se consideró al tipo de cambio, el cual es definido por Clement, Pool y Carrillo (1991) como "el valor de una moneda extranjera a precios de la moneda local, es decir, es la expresión de una moneda en términos de otra". En segundo lugar, se considera la tasa de interés, que de acuerdo a Samuelson y Nordhaus (2002), se puede entender como el precio del dinero, y que en nuestro estudio está representada por la tasa de fondeo gubernamental. Siguiendo la definición que, de esta tasa en específico, da el Banco de México (2016) en su página oficial: "la tasa representativa de las operaciones de crédito al mayoreo realizadas por la banca y casas de bolsa en el mercado interbancario utilizando como garantía títulos de deuda pública, a plazo de 1 día". Finalmente, la tercera variable a considerar, fue el precio del petróleo, mismo que es determinado por la oferta y demanda de este commodity con base en los acontecimientos internacionales.

Las variables anteriores fueron elegidas considerando, por un lado, su importancia e influencia como indicadores económico-financieros en las operaciones de trading cotidianas en los mercados financieros; y, por otro lado, la aparición de las mismas en la literatura financiera que trata de estudiar la relación entre las variables mencionadas. A continuación, se presenta una revisión de la literatura más relevante, donde se ha estudiado la relación de estas variables de forma individual o conjunta en los mercados accionarios de distintos países, así como, algunas investigaciones que han analizado la relación entre estas y otras variables macroeconómicas importantes.

En cuanto a la variable tipo de cambio, Blahun (2019) realiza un estudio cuyo objetivo es medir el impacto de variaciones en el tipo de cambio sobre el mercado accionario de Ucrania, descubriendo una causalidad bilateral entre los mismos. En el mismo sentido, Singh (2015) analiza la relación entre el tipo de cambio y los precios de las acciones en India, revelando que ambas variables están co-integradas, mostrando evidencia significativa del tipo de cambio como determinante del precio de las acciones y viceversa. Por otra parte, Hajilee y Al Nasser (2014), demuestran que, en China, Pakistán, México y Venezuela, la volatilidad del tipo de cambio tiene un importante efecto negativo sobre el desarrollo del mercado bursátil, mientras que en Filipinas y Sudáfrica afecta positivamente. Sin embargo, Živkov, Balaban y Djurašković (2018) tratan de explicar el nexo que existe entre las bolsas de valores y el tipo de cambio de los principales mercados emergentes de Europa y Asia, descubriendo que la correlación entre estos dos tipos de mercados no es particularmente fuerte en el periodo observado, con excepción del periodo de la crisis financiera mundial. En ese mismo sentido, Sinha y Kholi (2015) estudian el efecto del tipo de cambio sobre tres índices bursátiles de la Bolsa de Bombay, encontrando que no hay 
interacciones significativas entre el tipo de cambio, rupia india, y los rendimientos accionarios. Igualmente, Javaid y Kousar (2018) analizan el impacto de las fluctuaciones del tipo de cambio en la volatilidad del principal índice de la Bolsa de Irán, sin encontrar un impacto significativo.

En cuanto al efecto del tipo de interés, estudios recientes como el de El Abed y Zardoub (2019) revelan que el tipo de interés tiene un impacto negativo y significativo en los rendimientos accionarios en el mercado alemán. Por su parte, Stoica, Nucu y Diaconasu (2014) encuentran efectos significativos de las tasas de interés internacionales sobre los índices bursátiles de Republica Checa, Hungría, Polonia y Rumania. Por su parte, Hsing (2011) encuentra que el índice bursátil Lituano es afectado de forma negativa por la tasa de interés real doméstica y por el rendimiento de la deuda pública de la Zona Euro, y, posteriormente, Hsing y Hsieh (2012) revelan que el índice bursátil de Polonia se encuentra negativamente afectado por las mismas variables que el estudio anterior.

En cuanto al precio del petróleo, trabajos recientes, como el de Çetin y Altun (2019), investigan el impacto de los precios de petróleo de la OPEC en las bolsas de valores de Reino Unido y Estados Unidos, encontrando que los impactos de los precios del petróleo disminuyen los rendimientos accionarios de ambos mercados. Sin embargo, Wen et al. (2019) investigan la relación entre los precios del petróleo y el mercado accionario japonés, encontrando resultados empíricos que muestran que no hay efectos de co-integración asimétrica significativa entre los precios del petróleo y el mercado accionario chino, ni a niveles generales, ni a niveles sectoriales. En este mismo sentido, Karakas (2019) examina la estructura de dependencia de tres índices de la Bolsa de Estambul y el precio internacional del petróleo Brent, descubriendo una estructura de dependencia débil entre estas variables.

Por otro lado, existen estudios donde las variables expuestas para esta investigación son interrelacionadas para explicar la conexión existente entre ellas, destacándose los estudios que se enfocan en la relación tipo de cambio y precio del petróleo. En este sentido, Basnet, Vatsa y Sharma (2014) muestran como las fluctuaciones del precio del petróleo pueden afectar significativamente a los diversos sectores de la economía en países exportadores de este, afectando elementos que van desde el aumento de los costos de producción, hasta la existencia de una relación positiva en el largo plazo entre los precios del petróleo y el tipo de cambio real del dólar canadiense y la corona noruega. Por su parte, Méndez-Carbajo (2011) también plantea que los cambios del precio del petróleo son consecuencia importante de la oferta y la demanda de divisas, y agrega que esta relación aumenta con el grado de dependencia energética, como en el caso de la República Dominicana. Así mismo, Akram (2004) expone una relación no lineal negativa existente entre los precios del petróleo y los tipos de cambio en el caso de Noruega. Finalmente, Al-mulali y Che Sab (2012) consideran que en países exportadores de petróleo como Egipto, Nigeria, Arabia Saudita, Emiratos Árabes Unidos y Venezuela, el tipo de cambio depende, entre otras cosas, del precio del petróleo, ya que el aumento el precio de este provoca una revaluación en el tipo de cambio.

Por último, se encuentran también investigaciones que estudian el efecto de otras variables macroeconómicas en las bolsas de valores, por ejemplo, el de Sabäu-Popa, Bolos, Scarlat, Delcea y Bradea (2014) quienes concluyen que el PIB ha tenido una influencia significativa en el índice de referencia de la Bolsa de Valores de Bucarest, mientras que otros factores macroeconómicos solo apoyan el crecimiento económico sin lograr grandes variaciones en la bolsa.

\section{METODOLOGÍA}

EL IPC debe su variación a los cambios producidos por la Ley de la oferta y la demanda de las acciones, derivado de las decisiones que toman los inversionistas a partir de los factores que se dan dentro y fuera de las emisoras de acciones. Pero ¿En qué medida estos factores son capaces de modificar al IPC?

El objetivo general de la investigación es proponer variables macroeconómicas que permitan explicar el comportamiento del mercado accionario en México, a través de una investigación empírica y utilizando un contraste econométrico de corte transversal de datos históricos reales, que permitan definir la importancia e influencia de estos, sobre del IPC. Por tal motivo, se plantea como hipótesis:

$H_{0}=$ La variación del IPC depende de los cambios que sufre el tipo de cambio, la tasa de interés y el precio del petróleo.

Las variables independientes son: el tipo de cambio pesodólar, medido a través del tipo de cambio fix, calculado por el Banco de México; la tasa de interés, la cual es presentada por la tasa de fondeo gubernamental (calculada por el Banco de México) y el precio del petróleo Western Texas Intermediate -WTI-. Los datos abarcan desde el 02 de enero del 2008 hasta el 31 de diciembre del 2015 con una periodicidad mensual. El periodo utilizado en este estudio responde a la disponibilidad de la información en las 
fuentes consultadas. Los valores perdidos en las distintas series fueron tratados bajo el criterio de exclusión de casos según pareja. Es decir, solo se tomó en cuenta el número de observaciones en donde todas las variables tenían información, lo que produjo un total de 1896 observaciones para realizar el estudio empírico. El software utilizado para el tratamiento estadístico fue $I B M$ SPSS Statistics 23. Como es habitual en este tipo de estudios, todas las series históricas de precios se convirtieron a rendimientos logarítmicos para su análisis.

Para el proceso y análisis de los datos históricos se desarrolló: 1) un análisis de correlación lineal; 2) un contraste unifactorial por medio de regresión lineal simple; y 3 ) un contraste multifactorial a través de regresión lineal múltiple. Con estos análisis, primero, se determinó la relación e influencia de cada una de las variables independientes en la variable dependiente, para finalizar con un modelo que incluye todas las variables y mide la influencia que estas tienen sobre el IPC.

\section{Análisis de Correlación Lineal}

Lind, Mason y Marchal (2003) definen este análisis como "un grupo de técnicas para medir la magnitud de la relación entre dos variables" (p. 368). Para llevar a cabo este análisis se utiliza el coeficiente de correlación de Pearson, y se obtiene de la siguiente manera:

Donde:

$$
r=\frac{\sum(x-\bar{x})(y-\bar{y})}{(n-1) S_{X} S_{Y}}
$$

$r=$ Coeficiente de correlación

$\bar{x}=$ Media de la suma de las variables $x$.

$\bar{y}=$ Media de la suma de las variables $y$.

$n=$ Número de pares de observaciones.

$$
\begin{aligned}
& S_{X}=\text { Varianza variable } x \\
& \qquad \sqrt{\frac{\sum x^{2}-n \bar{x}^{2}}{n-1}} \\
& S_{Y}=\text { Varianza variable } y \\
& =\sqrt{\frac{\sum y^{2}-n \bar{y}^{2}}{n-1}}
\end{aligned}
$$

Los resultados pueden variar desde 0 a 1 , donde el cero (0) indica una correlación nula mientras que la unidad (1) representa una correlación perfecta. Además, el signo del resultado indica si se trata de una relación positiva o negativa.

Una manera de comprobar la confiabilidad del coeficiente de correlación, y determinar que realmente existe una correlación entre las variables es a través de la prueba $t$ (Valdez, 2016), la cual se inicia planteando las siguientes hipótesis:

$H_{0}: r=0$ Es decir, la correlación es cero.

$H_{1}: r \neq 0$ La correlación es diferente a cero.

En donde se generan dos posibles resultados, los cuales se obtienen con la siguiente formula:

$$
t=\frac{r \sqrt{n-2}}{\sqrt{1-r^{2}}}
$$

Donde:

$n-2=$ Grados de libertad, representados por gl.

El valor de significancia utilizado para esta investigación es del 0,05 ; si la hipótesis no se rechaza, la correlación entre variables es nula, mientras que, si se rechaza, se concluye una relación existente entre variables.

\section{Análisis de Regresión Lineal Simple}

Después de demostrar que las variables se relacionan entre sí y poseen un grado de correlación aceptable, se realiza un análisis de regresión lineal simple. Lind et al. (2003) describen el análisis de regresión lineal simple como una forma que permite representar la relación existente de dos variables cuantitativas en forma matemática, a través de una ecuación, la cual se denomina ecuación de regresión o ecuación de ajuste, y se representa de la siguiente forma:

$$
\hat{y}=\beta_{0}+\beta_{1} x+\varepsilon
$$

Donde:

$\hat{y}=$ Ajuste de la variable dependiente.

$\beta_{0}=$ Punto de intersección con el eje $y$ en el diagrama de dispersión, en este punto inicia la línea recta.

$\beta_{1}=$ Proporción que aumenta o disminuye de la variable dependiente por cada unidad de la variable independiente. (Pendiente de la recta).

$x=$ Variable independiente.

$\varepsilon=$ Error, que permite ajustar la línea. Residuo del modelo.

Para la estimación de los parámetros del modelo de regresión, se utiliza el método de mínimos cuadrados ordinarios (MCO), que según Góngora y Hernández (1999):

proporciona la línea de mejor ajuste al mismo tiempo que busca darle un valor a los coeficientes de regresión a través de la minimización de la 
suma de los cuadrados de la distancia entre los valores de la variable dependiente reales y los ajustados (p. 306).

Lo anterior se determina en base a las siguientes expresiones: ${ }^{1}$

$$
\begin{gathered}
\beta_{1}=b_{1}=\frac{n\left(\sum x y\right)-\left(\sum x\right)\left(\sum y\right)}{n\left(\sum x^{2}\right)-\left(\sum x\right)^{2}} \\
\beta_{0}=b_{0}=\frac{\sum y-\beta_{1} \sum x}{n}=\bar{y}-\beta_{1} \bar{x}
\end{gathered}
$$

Aun cuando se conoce que existe una correlación entre las variables, es necesario definir el coeficiente de determinación, el cual corresponde a $R^{2}$, ya que este "nos indica que tan confiable es predecir la variable dependiente en términos de la independiente" (Lind et al., 2003, p. 372). Es decir, determina el porcentaje en el que la variable independiente es capaz de explicar a la dependiente.

Siguiendo a Anderson, Sweeney y Williams (2008) la formulación para el cálculo del coeficiente de determinación es como sigue:

Donde:

$$
R^{2}=\frac{S C R}{S T C}
$$

$R^{2}=$ Coeficiente de determinación.

\section{SCR}

= Suma de cuadrados debida a la regresión

$=\sum\left(\widehat{y}_{l}-\bar{y}\right)^{2}$

STC = Suma total de cuadrados

$$
=\sum\left(y_{i}-\bar{y}\right)^{2}
$$

Sin embargo, también se utiliza el coeficiente de determinación ajustado, ya es una forma de precisar el modelo, cuando se adicionan un mayor número de variables explicativas. Según IBM Knowledge Center (2019) "el R cuadrado ajustado se calcula dividiendo el error cuadrático de la media residual por el error cuadrático promedio total (que es la varianza muestral)". Su formulación de acuerdo a Anderson et al. (2008) es la siguiente:

$$
\mathrm{R}_{\mathrm{a}}^{2}=1-\left(1-\mathrm{R}^{2}\right) \frac{\mathrm{n}-1}{\mathrm{n}-\mathrm{k}-1}
$$

Donde:

$R_{a}^{2}=$ Coeficiente de determinación ajustado.

$R^{2}=$ Coeficiente de determinación.

$\mathrm{n}=$ número de observaciones.

$\mathrm{k}=$ número de variables independientes.

Después se obtiene el valor estadístico de la prueba F, que también se utiliza para demostrar la significancia de la regresión del modelo de forma global. Según Anderson $e t$ al. (2008):

cuando sólo se tiene una variable independiente, la prueba $\mathrm{F}$ lleva a la misma conclusión que la prueba $t$; es decir, si la prueba $t$ indica que $\beta_{1} \neq 0$ y por lo tanto que existe una relación significante, la prueba $\mathrm{F}$ también indicará que existe una relación significante. Pero cuando hay más de una variable independiente, sólo la prueba $\mathrm{F}$ puede usarse para probar que existe una relación significante genera (p. 571).

\section{Análisis de Regresión Lineal Múltiple}

Según Rojo (2007), el análisis de regresión lineal múltiple permite no sólo explicar a la variable dependiente con la ayuda de una sola variable explicativa, sino con la ayuda de dos o más variables. El número de variables depende de la situación y el criterio del investigador. Este análisis pretende mostrar que una variable dependiente se ve influenciada por más de una variable independiente, con el fin de obtener estimaciones más precisas acerca de esta. La ecuación matemática que expresa la estimación de la variable dependiente en la población, es la siguiente:

$$
\hat{y}=\beta_{0}+\beta_{1} x_{1}+\beta_{2} x_{2}+\ldots+\beta_{k} x_{k}+\varepsilon
$$

Donde:

$\hat{y}=$ Ajuste de la variable dependiente.

$\beta_{0}=$ Punto de intersección con el eje $y$ en el diagrama de dispersión, en este punto inicia la línea recta.

$\beta_{1}, \beta_{2}, \ldots \beta_{1 k}=$ Proporción que aumenta o disminuye de la variable dependiente por cada unidad de la variable independiente.

$x=$ Variable independiente.

$\varepsilon=$ Error, que permite ajustar la línea. Residuo del modelo.

Dentro del análisis de regresión lineal múltiple se determina el coeficiente de correlación y determinación,

en este trabajo, dejando dicha comprobación y sus implicaciones para futuras investigaciones.
${ }^{1} \mathrm{Al}$ ser un primer estudio preliminar para evaluar la posible dependencia de las variables macroeconómicas utilizadas en esta investigación sobre el IPC de la $\mathrm{BMV}$, los supuestos básicos de la estimación mediante MCO, no se comprobaron 
los cuales miden, en conjunto, la relación y la influencia de las variables explicativas sobre la variable dependiente; entre más se acerque el resultado a la unidad (1), el nivel de correlación será mayor, lo que significa que las variables guardan relación.

En el caso de la regresión lineal múltiple, para llevar a cabo la prueba F (Lind et al., 2003), primero es necesario plantearse dos hipótesis, las cuales se expresan de la siguiente manera:

$H_{0}: \beta_{1}=\beta_{2}=\beta_{k}=0$

$H_{1}$ : No todas las $\beta$ son 0

La prueba $\mathrm{F}$ se realiza mediante la siguiente fórmula:

$$
F=\frac{S C R / k}{S C E /[n-(k+1)]}
$$

Donde:

$k=$ Número de variables independientes.

$n=$ Número de observaciones

$S C R$

= Suma de cuadrados debida a la regresión

$=\sum\left(\widehat{y_{l}}-\bar{y}\right)^{2}$

SCE $=$ Suma de cuadrados del error

$$
=\sum\left(y_{i}-\hat{y}\right)^{2}
$$

Para poder comprobar la confiablidad de los resultados se realiza la prueba $t$. Lind et al. (2003), proponen que aun cuando se ha establecido una correlación entre la variable dependiente y las independientes, es necesario realizar una prueba t para asegurarse que las variables independientes causan una variación significativa en la variable dependiente. En este proceso, se realiza una prueba de cada una de las variables independientes para determinar si existe una relación significativa con la variable dependiente.

Para este proceso se plantean dos hipótesis:

$$
H_{0}: B_{i}=0
$$

$H_{1}: B_{i} \neq 0$.

Y la fórmula a realizar es la siguiente:

$$
t=\frac{b_{i}}{s_{b_{i}}}
$$

Donde:

$b_{i}=$ Coeficiente de cualquiera de las variables independientes.

$s_{b_{i}}=$ Error estándar del coeficiente de regresión del que se trate. El cual se recomienda obtener a través de un programa especializado.

El resultado se compara con el obtenido de la tabla de distribución $t$ de Student con $n-k-1$ grados de libertad y con el nivel de significancia que se le hubiera determinado a la prueba.

Las estimaciones de los parámetros del modelo de regresión múltiple se realizaron igualmente mediante mínimos cuadrados ordinarios, con las limitaciones señaladas en la nota al pie precedente.

\section{RESULTADOS}

En este apartado, se utiliza la siguiente nomenclatura de las variables utilizadas: Índice de Precios y Cotizaciones (IPC); tipo de cambio (TC); tasa de interés (TI) y precio del petróleo (PP).

\section{Resultados del Análisis de Correlación}

En la Tabla 1, se presentan los resultados del análisis de correlación de Pearson con sus respectivos niveles de significación de la prueba $t$. En estos resultados se detecta una correlación considerable significativa y negativa en el caso del IPC con el tipo de cambio, así como una correlación moderada y positiva con el precio del petróleo. Para el tipo de interés el índice de correlación de Pearson es muy bajo y no significativo. Derivado de lo anterior se desprende que, al menos con dos de las variables explicativas planteadas pudiera existir una adecuada estructura de correlación, y se procedió a probar si existiera alguna relación de dependencia de acuerdo a la hipótesis planteada, mediante las técnicas de regresión lineal simple y múltiple. 
Tabla 1. Análisis de correlación: IPC, TC, TI y PP.

\begin{tabular}{|c|l|l|l|l|l|}
\hline \multicolumn{2}{|l|}{} & \multicolumn{1}{|c|}{ IPC } & \multicolumn{1}{|c|}{ TC } & \multicolumn{1}{|c|}{ PI } & \multicolumn{1}{|c|}{ PP } \\
\hline IPC & Correlación de Pearson & 1 & $-0,433^{* *}$ & $-0,008$ & $0,343^{* *}$ \\
\hline TC & $p$-value & Correlación de Pearson & $\mathbf{0 , 0 0 0}$ & 0,735 & $\mathbf{0 , 0 0 0}$ \\
\hline & $p$-value & $-0,433^{* *}$ & 1 & $-0,005$ & $-0,359^{* *}$ \\
\hline TI & Correlación de Pearson & $\mathbf{0 , 0 0 0}$ & & 0,813 & $\mathbf{0 , 0 0 0}$ \\
\hline & $p$-value & $-0,008$ & $-0,005$ & 1 & 0,001 \\
\hline PP & Correlación de Pearson & 0,735 & 0,813 & & 0,961 \\
\hline & $p$-value & $0,343^{* *}$ & $-0,359^{* *}$ & 0,001 & 1 \\
\hline$* *$ La correlación es significativa en el nivel 0,01 (bilateral). & & 0,961 & \\
\hline
\end{tabular}

Fuente: elaboración propia.

\section{Resultados del Análisis de Regresión Lineal Simple}

En esta sección se presentan los resultados de los análisis de regresión lineal simple donde se contrapuso cada una de las variables independientes planteadas contra el IPC. ${ }^{2}$

La Tabla 2 muestra un resumen de los modelos unifactoriales de regresión simple contrastados. Para cada uno de ellos se presenta su $\mathrm{R}^{2}$, su $\mathrm{R}^{2}$ ajustado, el valor del estadístico $\mathrm{F}$ de significación global del modelo y su $p$ value; así como el valor estimado de los coeficientes $\beta 0$ (constante) y $\beta 1$ (variable predictora) en cada modelo, con sus correspondiente $p$-values de la prueba t de significación estadística individual de cada parámetro estimado. Como se puede observar, solo los modelos que consideraron a las variables tipo de cambio y precio del petróleo respectivamente, produjeron coeficientes con significación estadística desde niveles del $1 \%$, tanto para el modelo en general, como para los parámetros correspondientes a las variables predictivas consideradas de forma individual. Sin embargo, en el caso del petróleo, el parámetro correspondiente a la constante tampoco fue significativo. Así mismo, el grado de explicación del modelo, dado por el coeficiente de determinación $\mathrm{R}^{2}$, tanto sin ajustar como ajustado, dieron valores muy bajos, por lo que de forma individual las variables tipo de cambio y petróleo, solo alcanzarían a explicar el comportamiento de la variable dependiente aproximadamente en un $18 \%$ por parte del TC y de un $10 \%$ tratándose del PP.

Tabla 2. Resumen de los modelos de regresión simple

\begin{tabular}{|l|ccccc|}
\hline & $\mathbf{R}^{2}$ & $\mathbf{R}_{\mathbf{a}}^{2}$ & $\mathbf{F}$ & $\boldsymbol{\beta}_{0}$ & $\boldsymbol{\beta}_{1}$ \\
\hline $\begin{array}{l}\text { Modelo 1: Variable independiente: Tipo de cambio. }\left(\boldsymbol{\beta}_{1}\right) \\
\text { p-values }\end{array}$ & 0,188 & 0,187 & 453,472 & 0,000 & $-0,745$ \\
\hline $\begin{array}{l}\text { Modelo 2: Variable independiente: Tasa de interés. }\left(\boldsymbol{\beta}_{1}\right) \\
\text { p-values }\end{array}$ & 0,000 & 0,000 & 0,114 & 0,000 & $-0,003$ \\
\hline $\begin{array}{l}\text { Modelo 3: Variable independiente: Precio del petróleo. }\left(\boldsymbol{\beta}_{1}\right) \\
\text { p-values }\end{array}$ & 0,108 & 0,108 & 250,269 & 0,000 & 0,169 \\
\hline
\end{tabular}

Fuente: elaboración propia en base a estimaciones realizadas.

${ }^{2}$ Dado el carácter preliminar de esta investigación sobre la relación y dependencia de las variables utilizadas, la comprobación de los supuestos del método de MCO (datos y residuos) para la estimación de los parámetros del modelo, quedan fuera del alcance de esta investigación. Por consiguiente, los resultados de este estudio deben verse bajo esta óptica. 
Centrándose en los dos modelos que tuvieron resultados estadísticamente significativos, sus correspondientes expresiones matemáticas del modelo de regresión lineal simple estimado serían las siguientes: en cuanto a la variable predictora tipo de cambio, la expresión sería: $\hat{y}=$ $-0,745 x$, donde el $-0,745$ representa la pendiente de la recta de regresión, y significa que, por cada punto de rendimiento que aumenta el TC, el IPC disminuye un 0,745 de punto. Respecto a la variable independiente precio de petróleo, se tendría: $\hat{y}=0,169 x$, donde el 0,169 representa el aumento de la variable dependiente por cada punto de rendimiento obtenido por el PP.

\section{Resultados del Análisis de Regresión Lineal Múltiple}

En este apartado se presentan los resultados del modelo multifactorial en que se consideraron las tres variables: tipo de cambio, tipo de interés y precio del petróleo, de manera conjunta en el mismo modelo, como predictoras del comportamiento del IPC. ${ }^{3}$

En la Tabla 3 se muestra un resumen de los resultados de dicho modelo, la cual presenta de manera análoga los mismos elementos que la tabla correspondiente a los resultados de la regresión lineal simple.

Tabla 3. Resumen de los modelos de regresión lineal múltiple

\begin{tabular}{|l|ccccccc|}
\hline & $\mathbf{R}^{\mathbf{2}}$ & $\mathbf{R}_{\mathbf{a}}^{2}$ & $\mathbf{F}$ & $\boldsymbol{\beta}_{0}$ & $\boldsymbol{\beta}_{1}$ & $\boldsymbol{\beta}_{2}$ & $\boldsymbol{\beta}_{3}$ \\
\hline $\begin{array}{l}\text { Modelo 3 variables independientes: } \mathbf{T C} \\
\left(\boldsymbol{\beta}_{1}\right) \text {, } \mathbf{T I}\left(\boldsymbol{\beta}_{2}\right), \mathbf{P P}\left(\boldsymbol{\beta}_{3}\right) .\end{array}$ & 0,224 & 0,223 & 197,6 & 0,000 & $-0,624$ & $-0,004$ & 0,104 \\
p-values & & & $\mathbf{0 , 0 0 0}$ & 0,079 & $\mathbf{0 , 0 0 0}$ & 0,615 & $\mathbf{0 , 0 0 0}$ \\
\hline
\end{tabular}

Fuente: elaboración propia basada en estimaciones realizadas.

El grado de explicación del modelo, considerando las tres variables de manera conjunta, aumenta a un $22 \%$, no pudiéndose rechazar la hipótesis nula de que de todos los parámetros del modelo son iguales a cero, por lo que se produce evidencia estadísticamente significativa en favor del modelo multifactorial de forma global, desde niveles del $1 \%$. Nuevamente, las únicas dos variables en las que se rechaza la hipótesis nula de que los valores individuales de sus coeficientes son cero (0), en términos estadísticamente significativos a los mismos niveles de confianza, son: el tipo de cambio y el precio del petróleo. No obstante, ahora en el modelo conjunto, para el caso de la constante, no se puede rechazar la hipótesis nula de que su valor sea cero (0).

Derivado de lo anterior, la ecuación de la regresión lineal múltiple de este modelo multifactorial, estaría formulada de la siguiente forma: $\hat{y}=-0,624 x_{1}+0,104 x_{3}$. Lo anterior implicaría que por cada punto de rendimiento que aumente el tipo de cambio, la variable dependiente disminuye en un 0,624 y que por cada punto de que aumente el precio del petróleo, el IPC aumentaría 0,104 de punto.

\section{DISCUSIÓN}

Los resultados obtenidos han mostrado cierta evidencia en favor de dos de las variables utilizadas, tanto en los modelos unifactoriales como en el modelo multifactorial aplicado: el tipo de cambio y el precio del petróleo. Siendo estos los posibles factores que explican el comportamiento del Índice de Precios y Cotizaciones de la Bolsa Mexicana de Valores, en el periodo analizado y bajo la metodología empleada en esta investigación.

Sin embargo, y dado el carácter preliminar de este estudio, los resultados deben verse bajo una óptica cautelosa, ya que la metodología de análisis econométrico empleada fue muy básica y ciertas limitantes que pudieran tener los resultados de la estimación, por la naturaleza misma de la técnica empleada, podrían hacer que los resultados no fueran del todo robustos. A pesar de esta limitación, se considera que la evidencia empírica encontrada, da indicios sobre la importancia de estas variables macroeconómicas utilizadas y proyecta futuras líneas de investigación que puedan reforzar o refutar los resultados aquí obtenidos.

\section{CONCLUSIÓN}

Los resultados de esta investigación representan una primera aproximación al entendimiento de los factores que explican el comportamiento del principal índice bursátil del mercado accionario mexicano. Para ello, se realizó un estudio empírico básico en el cual se utilizaron tres variables macroeconómicas principales, justificadas por; su importancia en el ámbito económico-financiero, en las 
operaciones de trading cotidianas y por los estudios científicos que han revelado su impacto en los mercados accionarios de diversos países y bajo distintas metodologías. La evidencia empírica obtenida nos hace concluir que, de acuerdo a la metodología empleada y las variables utilizadas en este estudio, el tipo de cambio y el precio del petróleo son factores que influyen en el comportamiento del Índice de Precios y Cotizaciones de la Bolsa Mexicana de Valores, en el periodo estudiado.

Algunas de las líneas futuras de investigación derivadas de los alcances y limitaciones de este trabajo podrían enfocarse en; primero, la comprobación de los supuestos relacionados con el método de estimación de mínimos cuadrados ordinarios, empleado para la estimación de los parámetros de los modelos de regresión, así como las implicaciones derivadas de los resultados de dichos contrastes. Segundo, la aplicación de una metodología de contraste econométrico bajo el enfoque de series de tiempo y ya no de corte transversal, o en su caso, la utilización de una metodología de datos panel, que combine las ventajas de ambos enfoques. Tercero, la aplicación de técnicas no paramétricas para los contrastes relacionados. Finalmente, la inclusión de otras variables macroeconómicas o la integración de otros enfoques aplicados en la teoría de modelos multifactoriales de valoración de activos financieros, distintos al de factores macroeconómicos, tales como: el técnico, el sectorial, el fundamental o el estadístico. En consecuencia, investigación adicional será necesaria para poder obtener mayor conocimiento sobre los factores que explican el comportamiento de los índices bursátiles en México y a nivel mundial.

\section{Declaración sobre conflictos de interés}

Las ideas planteadas en este documento, así como las opiniones, comentarios y conclusiones son responsabilidad de los autores de la misma y son independientes de su institución de afiliación. Así mismo durante la realización del trabajo y la redacción de este documento, no se ha incurrido en ningún conflicto de interés.

\section{REFERENCIAS BIBLIOGRÁFICAS}

Akram, Q. F. (2004). Oil prices and exchange rates:

Norwegian evidence. Econometrics Journal, 7(2), $\quad$ 476-504. doi:10.1111/j.1368423X.2004.00140.x

Al-mulali, U. y Che Sab, C. B. (2012). Oil prices and the real exchange rate in oil-exporting countries: a bounds testing approach. OPEC Energy Review, 36(4), 375-382. doi:10.1111/j.17530237.2012.00216.x

Anderson, D. R., Sweeney, D. J. y Williams, T. A. (2008). Estadística para la Administración y la Economía. México: Cengage Learning Editores. Banco de México. (2016). Tasa de fondeo gubernamental. Recuperado de http://www.banxico.org.mx/divulgacion/glosario /\#I

Basnet, H. C., Vatsa, P. y Sharma, S. (2014). Common Trends and Common Cycles in Oil Price and Real Exchange Rate. Global Economy Journal, 14(2), 249-263. doi: 10.1515/gej-20130042

Blahum, I. I. (2019). Causal Relationship between the Stock Market and Exchange Rate in Ukraine. The Problems of Economy, (1), 199-207. doi: https://doi.org/10.32983/2222-0712-2019-1-199207

Bolsa Mexicana de Valores. (2014). Índice de Precios y Cotizaciones (IPC). Recuperado de http://www.bmv.com.mx/docspub/INDICES/CTEN_INNM/NotaMetIPC_Sep2 014.pdf

Clement, N. C., Pool, C. y Carrillo, M.M. (1991). Economía Enfoque América Latina. México: Mc Graw Hill.

El Abed, R. y Zardoub, A. (2019). Exploring the nexus between macroeconomic variables and stock market returns in Germany: An ARDL Cointegration approach. Theoretical and Applied Economics, 2(2), 139-148. Recuperado de https://ideas.repec.org/a/agr/journl/vxxviy2019i2 (619)p139-148.html 
FACTORES QUE EXPLICAN EL COMPORTAMIENTO DEL MERCADO ACCIONARIO MEXICANO

Góngora Cortés, J. J. y Hernández Ramírez, R. (1999). Estadística Descriptiva. México: Trillas.

Hajilee, M. y Al Nasser, O. M. (2014). Exchange rate volatility and stock market development in emerging economies. Journal of Post Keynesian Economics, 37(1), 163-180. doi: https://doi.org/10.2753/PKE0160-3477370110

Hsing, Y. (2011). Macroeconomic variables and the stock market: the case of Lithuania. Review of Finance \& Banking, 3(1), 31-37.

Hsing, Y. y Hsieh, W. (2012). Impacts of macroeconomic variables on the stock market index in Poland: new evidence. Journal of Business Economics \& Management, 13(2), 334343. doi: https://doi.org/10.3846/16111699.2011.620133

IBM Knowledge Center. (2019). R2 ajustado. Recuperado de https://www.ibm.com/support/knowledgecenter/ es/SS4QC9/com.ibm.solutions.wa_an_overview. 2.0.0.doc/rsquared_adjusted.html

Karakas, M. (2019). An analysis of dependence between oil price and stock market with CopulaGARCH approach. An Empirical Analysis from Istanbul Stock Exchange. Thermal Science, 23(1), 33-46.

doi: https://doi.org/10.2298/TSCI180917328M

Lind, D. A., Mason, R. D. y Marchal, W. G. (2003). Estadística para administración y economía. México: McGraw-Hill.

Rojo, J. M. (2007). Regresión lineal múltiple. Recuperado de http://humanidades.cchs.csic.es/cchs/web_UAE/t utoriales/PDF/Regresion_lineal_multiple_3.pdf Méndez-Carbajo, D. (2011). Energy dependence, oil prices and exchange rates: The Dominican economy since 1990. Empirical Economics, 40(2), 509-520. doi: https://doi.org/10.1007/s00181-010-0340-4

Sabäu-Popa, D. C., Bolos, M. I., Scarlat, E., Delcea, C. y Bradea, I. (2014). Effects of macroeconomic variables on stock prices of the Bucharest stock Exchange (BSE). Economic Computation \& Economic Cybernetics Studies \& Research, 48(4), 98-108.

Samuelson, P. A. y Nordhaus, W. D. (2002). Macroeconomía con aplicaciones a Latinoamérica. España: Mc Graw Hill.

Singh, G. (2015). The Relationship between Exchange Rate and Stock Price in India: An Empirical Study. IUP Journal of Financial Risk Management, 12(2), 18-29.

Sinha, P., y Kohli, D. (2015). Modeling Exchange Rate Dynamics in India Using Stock Market Indices and Macroeconomic Variables. Amity Global Business Review, 105-18.

Stoica, O., Nucu, A. E. y Diaconasu, D.E. Interest Rates and Stock Prices: Evidence from Central and Eastern European Markets. Emerging Markets Finance and Trade, 50(4), 47-62. doi: https://doi.org/10.2753/REE1540496X5004S403

Valdez, I. P. (2016). Regresión Lineal Simple. Recuperado de http://www.dcb.unam.mx/profesores/irene/Notas /Regresion.pdf

Wen, F., Xiao, J., Xia, X., Chen, B., Xiao, Z. y Li, J. (2019). Oil Prices and Chinese Stock Market: Nonlinear Causality and Volatility Persistence. Emerging Markets Finance and Trade, 55(6), 
Gladys Iveth López-Juárez, Rogelio Ladrón de Guevara-Cortés y Rosa Marina Madrid-Paredones

$1247-1263$.

doi:

https://doi.org/10.1080/1540496X.2018.1496078

Živkov, D., Balaban, S. y Djurašković, J. (2018).

What Multiscale Approach Can Tell About the

Nexus Between Exchange Rate and Stocks in the
Major Emerging Markets? Czech Journal of Economics and Finance, 68(5), 491-512. Recuperado de https://ideas.repec.org/a/fau/fauart/v68y2018i5p 491-512.html 